\title{
Procalcitonin Strip Test as an Independent Predictor in Acute Pancreatitis
}

\author{
Brendan Hermenigildo Dias • Anthony Prakash Rozario • \\ Santosh Antony Olakkengil • Anirudh V.
}

Received: 17 December 2013 / Accepted: 23 May 2014 / Published online: 3 June 2014

(C) Association of Surgeons of India 2014

\begin{abstract}
Plasma procalcitonin (PCT) is a highly specific marker for the diagnosis of bacterial infection and sepsis. Studies have demonstrated its role in the setting of sepsis and acute pancreatitis. This study aims to analyze and compare the prognostic efficacy of plasma procalcitonin strip test in acute pancreatitis. A prospective study was conducted in the department of general surgery from June 2012 to June 2013. Plasma procalcitonin was estimated by the semiquantitative strip test. The study included a total of 50 patients diagnosed to have acute pancreatitis. Data was collected and statistically analyzed using SPSS version 17 . Thirty-nine out of the 50 patients $(78 \%)$ were males with a mean age of 46.8 years (range, $25-78$ years) and 25 patients $(50 \%)$ had ethanol-induced pancreatitis, while 13 patients $(26 \%)$ had gall stone pancreatitis. Plasma PCT values were found to correlate better than CRP levels and total leukocyte count with the total duration of hospitalization, ITU, and ICU stay, as well as with the progression to severe acute pancreatitis. A cut off for plasma PCT of $>2 \mathrm{ng} / \mathrm{mL}$ was found to be $100 \%$ sensitive and $100 \%$ specific and a cut off for CRP of $>19 \mathrm{mg} / \mathrm{dL}$ was $70 \%$ sensitive and $65 \%$ specific for predicting the progression to severe acute pancreatitis. Plasma PCT also correlated well with antibiotic requirement. A cut off value of $>0.5 \mathrm{ng} / \mathrm{mL}$
\end{abstract}

\section{B. H. Dias ( $ه)$}

Department of General Surgery, St. John's Medical College,

St. John's Mens Hostel, Sarjapura road, Koramangala,

Bangalore, India

e-mail: diasbrendan@gmail.com

\section{A. P. Rozario $\cdot$ S. A. Olakkengil $\cdot$ A. V.}

Department of General Surgery, St. John's Medical College

Hospital, Sarjapura road, Koramangala, Bangalore, Karnataka, India

A. P. Rozario

e-mail: rozarioa@yahoo.co.in

S. A. Olakkengil

e-mail: olakkengil@gmail.com

A. V.

e-mail: dranirudhv@gmail.com for plasma PCT was $100 \%$ sensitive and $80 \%$ specific and a cut off value of $>18 \mathrm{mg} / \mathrm{dL}$ for CRP was $86 \%$ sensitive and $63 \%$ specific for predicting antibiotic requirement. Plasma procalcitonin is an early and reliable prognostic indicator in acute pancreatitis. The procalcitonin strip test is a rapid test which is useful in analyzing prognosis in patients with acute pancreatitis.

Keywords Procalcitonin $\cdot$ Acute abdomen $\cdot$ Acute pancreatitis · C-reactive protein · CRP · PCT · Procalcitonin strip test

\section{Introduction}

Acute pancreatitis, defined as the acute nonbacterial inflammatory condition of the pancreas, is derived from the early activation of digestive enzymes found inside the acinar cells, with variable compromise of the gland itself, nearby tissues, and other organs. Most patients suffer a mild and limited disease but about one fifth of cases develop multiple organ dysfunction syndrome (MODS), accompanied by high mortality. Diagnosis is based on the presence of at least two of the following three features: abdominal pain; increased pancreatic amylase, and/or lipase levels to $\geq 3$ times the upper limit of normal; and imaging tests showing characteristic findings of acute pancreatitis [1].

Several inflammatory markers are being used routinely in various hospitals in India to assess the prognosis of patients with acute pancreatitis. Among these are the total and differential leukocyte counts, erythrocyte sedimentation rate, and CRP. Various scoring systems such as the RANSON scores have also been used to stratify patients with acute pancreatitis.

Plasma procalcitonin (PCT) is a highly specific marker for the diagnosis of bacterial infection and sepsis. Several studies on plasma PCT have demonstrated its role in the diagnosis of sepsis, prognosis of acute severe pancreatitis, and even as a prognostic marker following major surgery.[2-6] Beyond its value for the diagnosis of sepsis, PCT has also proved to be 
useful in monitoring the course and severity of the systemic inflammatory response. There are noninfectious conditions causing elevation in PCT levels. These include the following:

1. The first day(s) after

(a) A major trauma

(b) Major surgical intervention

(c) Severe burns

(d) Treatment with OKT3 antibodies and other drugs stimulating the release of proinflammatory cytokines

(e) Birth in neonates (age less than $48 \mathrm{~h}$ )

2. Patients with

(a) Prolonged or severe cardiogenic shock

(b) Prolonged severe organ perfusion abnormalities

(c) Small cell lung cancer or medullary $\mathrm{C}$ cell carcinoma of the thyroid

Most of the studies on PCT have focused mainly on patients with sepsis in the ICU setting and on patients with acute pancreatitis. Information regarding the role of PCT as a marker of acute inflammation in our population is grossly inadequate. It is an established fact that ethnic and racial variations exist among various population groups even when it comes to interpretation of serological tests. Thus, Western data regarding these serological markers may not be applicable to our population. Hence, it is important to have data regarding the levels of various serological markers of inflammation in our own population so that they can be used routinely as diagnostic adjuncts, indicators for intervention, and prognostic markers for patients presenting with acute abdomen in our part of the world.

Initiating a step in this direction, this study aims to analyze the prognostic efficacy of plasma procalcitonin in patients presenting with acute pancreatitis and compare it with other prognostic indicators.

\section{Materials and Methods}

Source of Data

The sources of data were all patients presenting with acute pancreatitis to the emergency medicine department of St. John's Medical College Hospital, Bangalore from June 2012 to June 2013.

\section{Sample Size}

A total of 50 patients were included in the study. Institutional Ethical Review Board (IERB) clearance was obtained for the study.

\section{Exclusion Criteria}

1. History of trauma

2. Prolonged cardiogenic shock with impaired organ perfusion

3. Lung cancer or medullary carcinoma of the thyroid

Study Design

Observational study—prospective study design

Protocol of the Procedure

1. Inclusion and exclusion criteria were applied to all patients presenting with acute pancreatitis to the emergency medicine department of our hospital.

2. Patients were educated about the study and only those patients consenting to participate in the study were included.

3. Database collection included documentation of medical history, age, sex, prehospital interval, vital signs, abdominal signs, and drug history.

4. CRP and plasma procalcitonin level determination was performed on the same serum sample drawn for other biochemical tests. The drawn blood was put in different vacutainers and labeled accordingly for the different tests. If there is not enough serum for performing CRP and plasma procalcitonin level determination, then that sample was to be discarded and a fresh sample was drawn.

5. Plasma procalcitonin was estimated using a semi quantitative strip test (B.R.A.H.M.S. PCT-Q test).

6. Descriptive and inferential statistical analysis was carried out on the data collected using SPSS 17.

\section{Results}

Out of the total of 50 patients, 39 patients $(78 \%)$ were males and 11 were females with a mean age of 46.8 years (range, 2578 years). Twenty-five patients ( $50 \%$ ) presented within $24 \mathrm{~h}$ of onset of symptoms. Twenty-seven patients (54\%) had recurrent pancreatitis and 26 patients ( $52 \%$ ) gave history of consumption of alcohol within $72 \mathrm{~h}$ prior to presentation. In 25 patients ( $50 \%$ ), ethanol abuse was found to be the cause for pancreatitis, while 13 patients $(26 \%)$ had gallstone pancreatitis. In 10 patients $(20 \%)$, no etiological factor was found and these patients were diagnosed to have acute idiopathic pancreatitis.

Majority of the patients (44\%) had high CRP levels between $9-20 \mathrm{mg} / \mathrm{dL}$ (normal value $<0.3 \mathrm{mg} / \mathrm{dL}$ ). However, contrastingly $46 \%$ of the patients had procalcitonin levels of $<0.5 \mathrm{ng} / \mathrm{mL}$ (normal value $<0.5 \mathrm{ng} / \mathrm{mL}$ ). Twenty-one patients (42\%) had RANSON score of 1 at admission. Four patients 
Table 1 Correlation coefficients for hospitalization

\begin{tabular}{llllr}
\hline Correlation coefficient & & Hospital stay $(P)$ & ITU stay $(P)$ & ICU stay $(P)$ \\
\hline Pearsons coefficient & PCT & $0.892(<0.001)$ & $0.745(<0.001)$ & $0.643(<0.001)$ \\
& CRP & $0.450(0.001)$ & $0.502(<0.001)$ & $0.097(0.501)$ \\
& WBC & $0.398(0.004)$ & $0.583(<0.001)$ & $-0.116(0.422)$ \\
\hline
\end{tabular}

developed organ dysfunction (MODS) requiring ICU care and two patients required ventilator support. There were no mortalities. A total of 21 patients (42\%) received antibiotics and of these 18 patients $(36 \%)$ received antibiotics for more than a week. In 10 patients $(20 \%)$, the indication was severe acute pancreatitis, while 11 patients received antibiotics in view of persistent fever for $>72 \mathrm{~h}$ after admission.

Total Leukocyte Count, CRP, and PCT Levels Versus Hospital Stay

As can be seen from Table 1, there was a strong positive correlation between plasma PCT levels and total hospital stay as well as intensive treatment unit (ITU) and ICU stay. Plasma PCT correlated better than CRP levels and total leukocyte count for total hospital stay and as well as ITU stay. Only Plasma PCT levels and not CRP or leukocyte count was found to correlate significantly with ICU requirement $(P<0.01)$.

Total Leukocyte Count, CRP, and PCT Levels Versus Antibiotic Requirement

Table 2 shows the strength of the correlation between total leukocyte count, CRP and PCT levels, and the requirement of antibiotics in pancreatitis. Only plasma PCT and CRP levels were found to correlate significantly $(P<0.01)$ with antibiotic requirement. Plasma PCT was found to correlate better than CRP levels for patients requiring antibiotics in acute pancreatitis.

Figure 1 shows the receiver operating characteristic (ROC) curves for antibiotic use. Using ROC curves, a cut off value of $>0.5 \mathrm{ng} / \mathrm{mL}$ at admission for plasma procalcitonin was $100 \%$ sensitive and $80 \%$ specific for predicting antibiotic requirement in patients with pancreatitis. Similarly, a cut off value of $>18 \mathrm{mg} / \mathrm{dL}$ for CRP was $86 \%$ sensitive and $63 \%$ specific for predicting antibiotic requirement. Total leukocyte count and
RANSON score at admission were not satisfactory in predicting antibiotic requirement.

Severe Acute Pancreatitis

A total of 10 patients $(20 \%)$ were diagnosed to have severe acute pancreatitis. All 10 patients underwent contrast enhanced computed tomography of the abdomen. The mean CT severity index in these patients was 5 (range, 3-8). Table 3 shows the correlation between plasma PCT and CRP levels and RANSON score and the severity of pancreatitis.

In our study, it was found that the RANSON score at admission as well as the CRP levels showed a positive correlation with the development of severe acute pancreatitis $(P<0.05)$. However, plasma PCT was found to correlate better than CRP levels and RANSON score in predicting the progression to severe acute pancreatitis (Table 3).

Figure 2 shows the receiver operating characteristic (ROC) curves for severe acute pancreatitis. Using ROC curves, a cut off value of $>2.0 \mathrm{ng} / \mathrm{mL}$ at admission for plasma procalcitonin was $100 \%$ sensitive and $100 \%$ specific for predicting the progression to severe acute pancreatitis. Similarly, a cut off value of $>19 \mathrm{mg} /$ $\mathrm{dL}$ for CRP was $70 \%$ sensitive and $65 \%$ specific for predicting severe acute pancreatitis. RANSON score at admission was specific but not sensitive in predicting severity of pancreatitis.

\section{Discussion}

In our study, we analyzed the role of total leukocyte counts, CRP and plasma procalcitonin levels, and the RANSON score at admission in predicting the prognosis, requirement of antibiotics, and predicting the progression to severe acute pancreatitis. We found a strong correlation between plasma PCT at admission and total hospital stay as well as requirement of ICU and ITU care. Plasma PCT values correlated better than
Table 2 Correlation coefficients for antibiotic requirement

$P<0.01$ is significant

\begin{tabular}{llll}
\hline Correlation coefficient & & Antibiotic requirement $(P)$ & Number of days of antibiotics $(P)$ \\
\hline Pearsons coefficient & PCT & $0.738(<0.001)$ & $0.852(<0.001)$ \\
& CRP & $0.503(<0.001)$ & $0.471(0.001)$ \\
& WBC & $0.222(0.121)$ & $0.298(0.035)$ \\
\hline
\end{tabular}


Fig. 1 Receiver operating characteristic $(R O C)$ curves for antibiotic use

\section{ROC Curve}

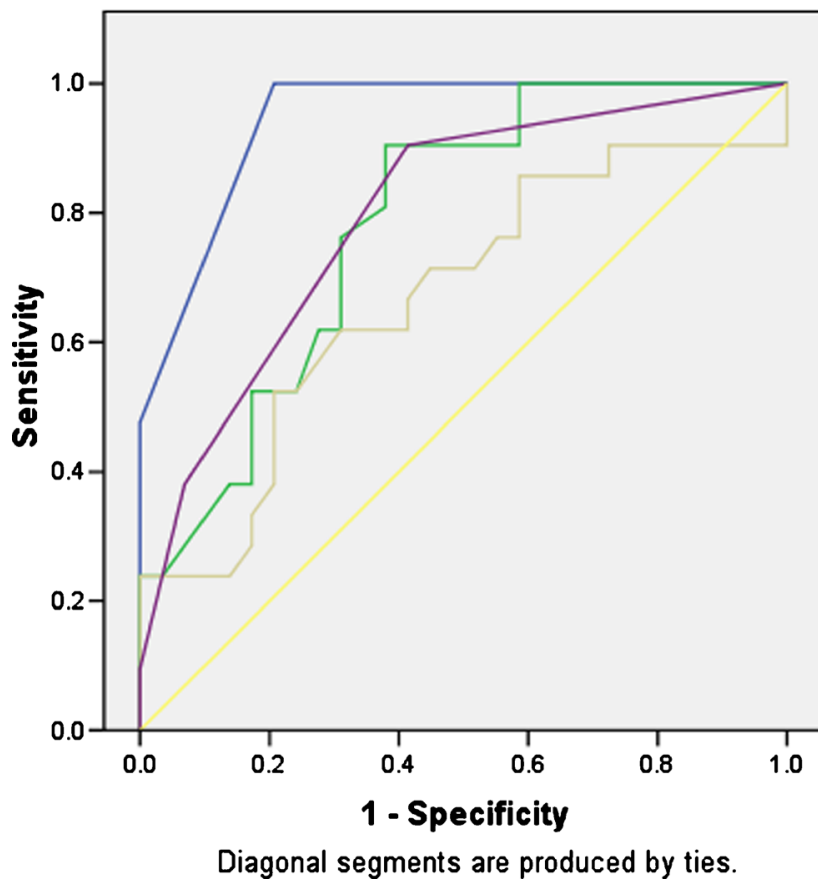

Source of the Curve

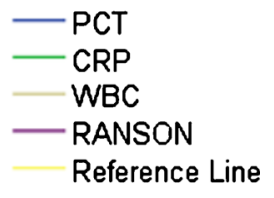

CRP and total leukocyte count in predicting the requirement of intensive care in patients presenting with acute pancreatitis (Table 1). Plasma PCT values were also found to correlate well with antibiotic requirement (Table 2) and was also found to be able to predict the progression to severe acute pancreatitis and organ failure (Table 3 ).

A large number of studies have assessed the role of plasma PCT and compared it to other inflammatory markers in predicting the severity of pancreatitis and the development of infected necrosis [7-14]. These studies have shown that plasma procalcitonin is a good marker for predicting severity and development of organ failure in acute pancreatitis and as well as predicting the development of infected pancreatic necrosis.

A prospective international multicenter study by Bettina $M$ et al. [15] assessed the role of plasma PCT in the development of pancreatic infections and overall prognosis of severe acute pancreatitis. In their study, they monitored both plasma PCT and CRP values routinely and concluded that monitoring of plasma procalcitonin allows early and reliable assessment of clinically relevant infections and overall prognosis in acute

Table 3 Correlation coefficients for severe acute pancreatitis

\begin{tabular}{lll}
\hline Correlation coefficient & & Severe acute pancreatitis $(P)$ \\
\hline Pearsons coefficient & PCT & $0.837(<0.001)$ \\
& CRP & $0.396(0.004)$ \\
& RANSON & $0.327(0.02)$ \\
\hline
\end{tabular}

$P<0.05$ is significant pancreatitis and thereby contributed to improved stratification of patients at risk to develop major complications.

In our study, we used a rapid, semi quantitative procalcitonin strip test (PCT-Q) for the estimation of plasma procalcitonin levels. We found that PCT-Q test was more accurate in predicting severe acute pancreatitis than CRP, with a cut off of $>2 \mathrm{ng} / \mathrm{mL}$ at admission being $100 \%$ sensitive and $100 \%$ specific for predicting severe acute pancreatitis. Another study by Kylänpää-Bäck et al. [16] also used PCT-Q test in the early detection of severe acute pancreatitis. They found that the PCT$\mathrm{Q}$ test was more accurate in predicting severe acute pancreatitis (sensitivity $92 \%$ and specificity $84 \%$ at $24 \mathrm{~h}$ ) than CRP, APACHE II score, and Ranson score. Its negative predictive value was high (97\% at $24 \mathrm{~h}$ ), and it detected each patient who developed subsequent organ failure.

A similar study by A. Olah et al.[17] assessed the role of PCT-Q strip test in differentiating sterile and infected forms of acute pancreatitis and found that PCT-Q test is a possible noninvasive method which can be used prior to or instead of fine-needle aspiration. They observed that elevated levels of procalcitonin (higher than $2 \mathrm{ng} / \mathrm{mL}$ ) clearly suggest infection, while lower values do not exclude the possibility of local sepsis.

Plasma PCT is an excellent marker of sepsis. However, many noninfectious, proinflammatory states such as major trauma, drugs stimulating release of proinflammatory cytokines, prolonged cardiogenic shock, and prolonged organ hypoperfusion can all cause raised plasma levels of PCT. Pancreatitis, if severe, can also cause massive release of proinflammatory cytokines with a corresponding rise in plasma PCT levels. Studies have proved that this rise in plasma PCT 
Fig. 2 Receiver operating characteristic $(R O C)$ curves for severe acute pancreatitis

\section{ROC Curve}

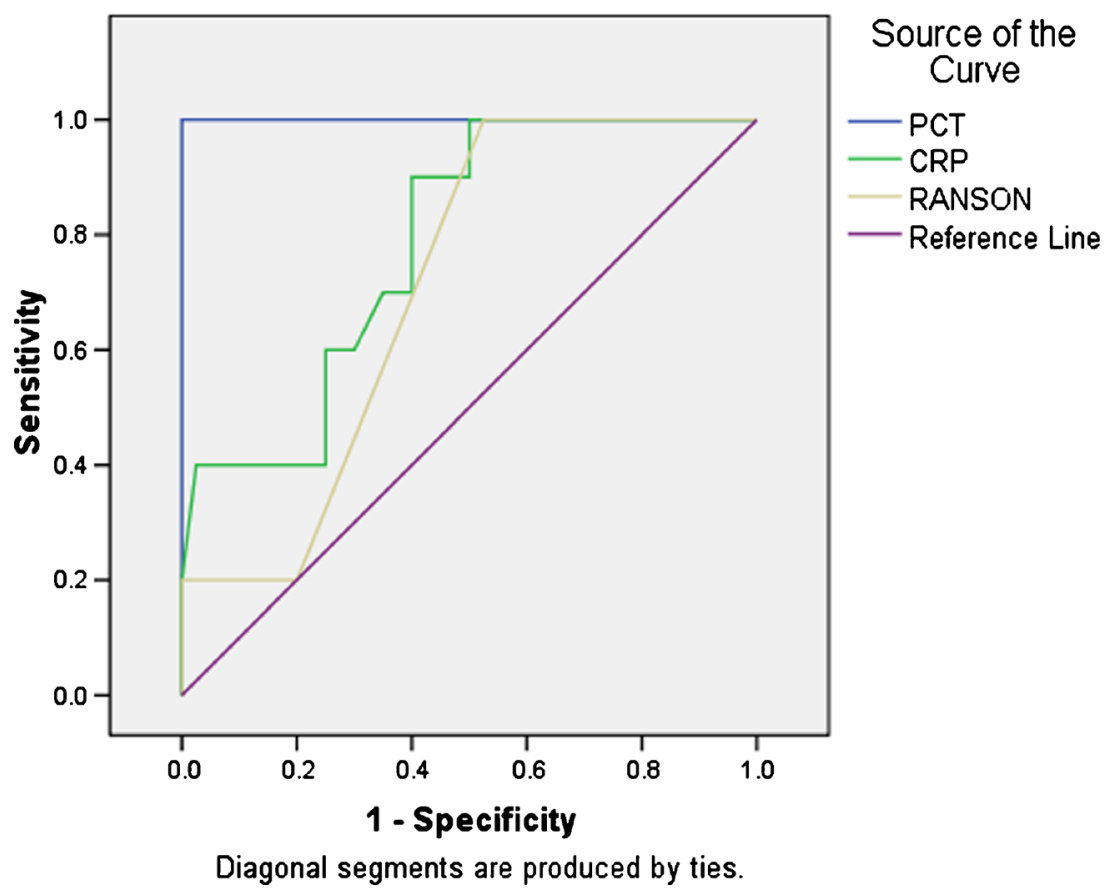

values is a predictor of disease severity. A meta-analysis by Mofidi R et al. [18] assessed the role of plasma procalcitonin in predicting the development of infected pancreatic necrosis and as well as the overall prognosis in noninfected severe acute pancreatitis. They concluded that plasma procalcitonin has a role in the management of both infected as well as noninfected severe acute pancreatitis. The meta-analysis also showed that the plasma PCT value within $24 \mathrm{~h}$ of admission can be used as a predictor of prognosis and subsequent values predict the development of infected pancreatic necrosis.

\section{Conclusions}

In patients with acute pancreatitis, plasma procalcitonin level at admission accurately identifies patients likely to progress to severe acute pancreatitis. Plasma procalcitonin at admission also helps predict antibiotic requirement and is found to be a better prognostic indicator than CRP levels and RANSON score in patients with acute pancreatitis.

Procalcitonin strip test is a rapid, useful, and accurate test in the risk stratification of patients presenting with acute pancreatitis.

\section{References}

1. Bradley EL (1993) A clinically based classification system for acute pancreatitis: summary of the international symposium on acute pancreatitis, Atlanta, Ga; September 11-13, 1992. Arch Surg 128:586-590

2. Simon L, Gauvin F, Amre DK et al (2004) Serum procalcitonin and $\mathrm{C}$-reactive protein levels as markers of bacterial infection: a systemic review and meta-analysis. Clin Infect Dis 39:206-217

3. Rau BM, Kemppainen EA, Gumbs AA, Buchler MW, Wegscheider K, Bassi C, Puolakkainen PA, Beger HG (2007) Early assessment of pancreatic infections and overall prognosis in severe acute pancreatitis by procalcitonin (PCT): a prospective international multicenter study. Ann Surg 245:745-754

4. Maruna P, Frasko R, Gurlich R (2008) Plasma procalcitonin in patients with ileus relations to other inflammatory parameters. Physiol Res 57:481-486

5. Assicot M, Gendrel D, Carsin H, Raymond J, Guilbaud J, Bohuon C (1993) High serum procalcitonin concentrations in patients with sepsis and infection. Lancet 341:515-518

6. Maruna P, Gurlich R, Frasco R, Chachkhiani I, Marunova M, Owen K, Peskova M (2002) Procalcitonin in the diagnosis of postoperative complications. Sb Lek 103:283-295

7. Kylänpää-Bäck M-L, Takala A, Kemppainen EA, et al. Procalcitonin, soluble interleukin-2 receptor, and soluble E-selectin in predicting the severity of acute pancreatitis. Critical Care Medicine. 2001;29(1)

8. Mándi Y, Farkas G, Takács T, Boda K, Lonovics J (2000) Diagnostic relevance of procalcitonin, IL-6, and sICAM-1 in the prediction of infected necrosis in acute pancreatitis. Int J Pancreatol 28(1):41-49

9. Modrau IS, Floyd AK, Thorlacius-Ussing O (2005) The clinical value of procalcitonin in early assessment of acute pancreatitis. Am J Gastroenterol 100(7):1593-1597

10. Mofidi R, Suttie SA, Patil PV, Ogston S, Parks RW (2009) The value of procalcitonin at predicting the severity of acute pancreatitis and development of infected pancreatic necrosis: systematic review. Surgery 146(1):72-81

11. Muller C, Uhl W, Printzen G et al (2000) Role of procalcitonin and granulocyte colony stimulating factor in the early prediction of infected necrosis in severe acute pancreatitis. Gut 46(2):233-238

12. Rau B, Steinbach G, Gansauge F, Mayer J, Grunert A, Beger H (1997) The potential role of procalcitonin and interleukin 8 in the prediction of infected necrosis in acute pancreatitis. Gut 41(6):832840 
13. Riché FC, Cholley BP, Laisné M-JC et al (2003) Inflammatory cytokines, $\mathrm{C}$ reactive protein, and procalcitonin as early predictors of necrosis infection in acute necrotizing pancreatitis. Surgery 133(3):257-262

14. Riché FC, Cholley BP, Laisné M-JC et al (2003) Inflammatory cytokines, $\mathrm{C}$ reactive protein, and procalcitonin as early predictors of necrosis infection in acute necrotizing pancreatitis. Surgery 133(3):257-262

15. Rau BM, Kemppainen EA, Gumbs AA, Buchler MW, Wegscheider K, Bassi C, Puolakkainen PA, Beger HG (2007) Early assessment of pancreatic infections and overall prognosis in severe acute pancreatitis by procalcitonin (PCT): a prospective international multicenter study. Ann Surg 245:745-754
16. Kylänpää-Bäck M-L, Takala A, Kemppainen E, Puolakkainen P, Haapiainen R, Repo H (2001) Procalcitonin strip test in the early detection of severe acute pancreatitis. Br J Surg 88(2): 222-227

17. Olah A, Belagyi T, Issekutz A, MAKAY R, Zaborszky A. Value of procalcitonin quick test in the differentiation between sterile and infected forms of acute pancreatitis. Hepatogastroenterology. 52(61):243-245

18. Mofidi R, Suttie SA, Patil PV, Ogston S, Parks RW (2009) The value of procalcitonin at predicting the severity of acute pancreatitis and development of infected pancreatic necrosis: systematic review. Surgery $146: 72-81$ 